OPEN ACCESS

Edited by:

Soumitra Paul Chowdhury, Helmholtz-Gemeinschaft Deutscher

Forschungszentren (HZ), Germany

Reviewed by:

Eric Boncompagni,

Université Côte d'Azur, France

Murali Gopal,

Central Plantation Crops Research Institute (ICAR), India

Youry Pii,

Free University of Bozen-Bolzano, Italy

*Correspondence:

Amanda Rosier

amandaro@udel.edu

Specialty section: This article was submitted to

Microbial Symbioses,

a section of the journa

Frontiers in Microbiology

Received: 19 August 2020 Accepted: 15 December 2020

Published: 15 January 2021

Citation:

Rosier A, Beauregard PB and Bais HP (2021) Quorum Quenching Activity of the PGPR Bacillus subtilis UD1022 Alters Nodulation Efficiency of Sinorhizobium meliloti on Medicago

truncatula.

Front. Microbiol. 11:596299 doi: 10.3389/fmicb.2020.596299

\section{Quorum Quenching Activity of the PGPR Bacillus subtilis UD1022 Alters Nodulation Efficiency of Sinorhizobium meliloti on Medicago truncatula}

\author{
Amanda Rosier ${ }^{1,2 *}$, Pascale B. Beauregard ${ }^{3}$ and Harsh P. Bais ${ }^{1,2}$ \\ ${ }^{1}$ Department of Plant and Soil Sciences, University of Delaware, Newark, DE, United States, ${ }^{2}$ Delaware Biotechnology \\ Institute, University of Delaware, Newark, DE, United States, ${ }^{3}$ Département de Biologie, Université de Sherbrooke, \\ Sherbrooke, QC, Canada
}

Plant growth-promoting rhizobacteria (PGPR) have enormous potential for solving some of the myriad challenges facing our global agricultural system. Intense research efforts are rapidly moving the field forward and illuminating the wide diversity of bacteria and their plant beneficial activities. In the development of better crop solutions using these PGPR, producers are including multiple different species of PGPR in their formulations in a "consortia" approach. While the intention is to emulate more natural rhizomicrobiome systems, the aspect of bacterial interactions has not been properly regarded. By using a tri-trophic model of Medicago truncatula A17 Jemalong, its nitrogen (N)-fixing symbiont Sinorhizobium meliloti Rm8530, and the PGPR Bacillus subtilis UD1022, we demonstrate indirect influences between the bacteria affecting their plant growthpromoting activities. Co-cultures of UD1022 with Rm8530 significantly reduced Rm8530 biofilm formation and downregulated quorum sensing (QS) genes responsible for symbiotically active biofilm production. This work also identifies the presence and activity of a quorum quenching lactonase in UD1022 and proposes this as the mechanism for non-synergistic activity of this model "consortium." These interspecies interactions may be common in the rhizosphere and are critical to understand as we seek to develop new sustainable solutions in agriculture.

Keywords: PGPR, symbiosis, consortia, legume, quorum sensing, quorum quenching, nodule, agriculture

\section{INTRODUCTION}

Legume crops are an essential component of sustainable agriculture due to their multifaceted benefits to ecology and human health (Stagnari et al., 2017). This is attributable to the mutualism between symbiotic $\mathrm{N}$-fixing bacteria (Rhizobia) and their specific legume plant hosts, referred to as "biological nitrogen fixation" (BNF). Rhizobia fix atmospheric $\mathrm{N}$ in exchange for carbon-rich photosynthates within specialized structures formed on the plant root called nodules (Oldroyd, 2013). Peoples et al. (2009) estimates that $30-40 \mathrm{~kg}$ of $\mathrm{N}$ is fixed per ton of crop legume dry matter and Herridge et al. (2008) approximates that $\mathrm{N}$-fixation by crop and forage legumes via symbiosis 
globally is roughly $50 \mathrm{Tg}$ per year. The ability of Rhizobia to fix nitrogen within an agricultural setting is a key factor in de-coupling dependence on synthetic nitrogen application. However, for BNF to effectively replace $\mathrm{N}$-fertilization, a clear understanding of the numerous mechanisms increasing $\mathrm{BNF}$ efficiency is required.

The interspecies signaling pathway between legumes and their bacterial symbionts responsible for BNF is well described, especially in the model legume Medicago truncatula, which is closely related to the forage crop alfalfa. Symbiosis between M. truncatula and Sinorhizobium meliloti commences through root exudation of the signaling plant flavonoid luteolin, which acts as a chemoattractant (Hassan and Mathesius, 2012). Luteolin induces transcription and expression of $S$. meliloti nod genes, producing lipo-chitooligosaccharide signals termed Nod factors (NFs) (Kondorosi et al., 1989). Receptors localized at the root hair recognize these NFs, instigating bacterial invasion and nodule organogenesis (Oldroyd et al., 2011; Gourion et al., 2015; Zipfel and Oldroyd, 2017); S. meliloti then divide, proliferate, and express $\mathrm{N}_{2}$-fixing nitrogenase enzyme within the plantderived nodules (Oldroyd, 2013). Legume symbiosis clearly relies on a finely tuned system of molecular pathways between bacteria and host.

For example, a key factor for the successful initiation of nodulation is the production of exopolysaccharides (EPS) by S. meliloti (Marketon et al., 2003). EPS include succinoglycan as well as high- and low-molecular-weight molecules of galactoglucan (EPS II) present in S. meliloti biofilms (Rinaudi and Gonzalez, 2009). The EPS II fraction within these biofilms are described as "symbiotically active"; EPS II defective mutants are unable to form pink nodules (González et al., 1996). EPS II production is dependent on the quorum sensing (QS) regulatory network including $\exp R$ (Pellock et al., 2002) and sinI genes (Marketon et al., 2003). The S. meliloti ExpR/SinI QS system relies on SinI synthase-produced long-chain $\mathrm{N}$-acyl homoserine lactone (AHL) signal molecules (Marketon et al., 2002; Gao et al., 2005). The AHL-bound ExpR protein controls the expression of $\sim 500$ QS genes (Gurich and González, 2009). One of these genes of note, $w g g R$, encodes a transcriptional regulator activating the downstream wge operons responsible for the biosynthesis and polymerization of EPS II low-molecularweight galactoglucans, the symbiotically active EPS component of S. meliloti biofilm (Gao et al., 2012). This QS pathway is a highly controlled intraspecies mode of communication that is crucial for $S$. meliloti to successfully coordinate activities at a community level. Considering these and the other myriad molecular communications occurring in the rhizosphere, it is imperative to inquire how other bacteria may be influencing these core symbiotic pathways.

Indeed, millions of different species of bacteria inhabit the ecosystem on and around plant roots, termed the "rhizosphere" (Adesemoye et al., 2009; Gamalero and Glick, 2011). Organisms within the rhizosphere improving plant health and resilience directly or indirectly are known as plant growth-promoting rhizobacteria (PGPR) (Kloepper et al., 1980; Pii et al., 2015). Many bacteria have been identified as generalist PGPRs for their association with a broad range of plants, and their specific plant beneficial activities have been described. Bacillus species are highly researched generalist PGPRs known to promote plant growth through their ability to solubilize nutrients and produce phytohormones, antifungal secondary metabolites, and volatile organic compounds (VOCs) (Aloo et al., 2019). Several Bacillus species are also known to express quorum quenching (QQ) enzymes, disrupting QS signaling of other bacteria, including pathogens (Dong et al., 2001; d'Angelo-Picard et al., 2005; Ryan et al., 2009). These activities are promising areas of rhizomicrobiome research as they may have unexpected influences on complex interspecies interactions.

Due to their numerous modes of action, PGPRs such as Bacillus have been utilized for crop applications and are a growing proportion of agrochemical company research efforts, where they are broadly termed "biologicals" (Timmusk et al., 2017; Marrone, 2019). Biological global markets are expanding (Arora et al., 2020), and the potential of these products is driving new market formulations incorporating multiple different species of live bacteria in "consortia" (Marrone, 2019). The rationale behind this innovation stems from the knowledge that natural rhizomicrobiomes are occupied by millions of different species of bacteria working in conjunction with one another (Schlatter et al., 2015); restoration of these microbial ecosystems may provide more robust benefits to the plant (Gouda et al., 2018; Sergaki et al., 2018). This concept, described as "synergism", manifests as "additive" plant benefits observed when multiple PGPRs are applied as compared to a single PGPR.

Synergistic plant growth promotion by multiple PGPR species has been observed in certain plant-bacteria-bacteria interspecies systems (Schwartz et al., 2013; Morel et al., 2015; Berendsen et al., 2018) but has also failed to produce in others (Felici et al., 2008; Kang et al., 2014; Maymon et al., 2015). Various co-inoculation combinations of Bacillus species and Rhizobia on legumes have shown synergistic growth and nodulation outcomes. Most prominently, nodulation was significantly enhanced in soybean (Glycine max L. Merr) systems when Bradyrhizobium japonicum was co-inoculated with $B$. cereus UW85 (Halverson and Handelsman, 1991), B. thuringiensis NEB17 (Bai et al., 2003), or B. amyloliquefaciens strain LL2012 (Masciarelli et al., 2014). B. subtilis-specific co-inoculations have been successful when used with Rhizobium leguminosarum bv. viciae 128C53 (Rlv) onto Pisum sativum L. (pea) (Schwartz et al., 2013) and when applied along with B. japonicum on soybean (Bai et al., 2003). No direct mechanisms of interaction were queried in these studies. Identifying suitable PGPR consortia requires understanding the multitude of plant beneficial activities that may be altered when the organisms coexist in what is now more commonly being described as the plant holobiont (ZilberRosenberg and Rosenberg, 2008). The lack of identified PGPR interspecies interaction mechanisms remains a significant gap in our knowledge, yet poses an opportunity to pursue empirical selections of appropriate PGPRs as we continue to expand our understanding of their plant beneficial activities.

To investigate meaningful legume-PGPR mechanisms, we designed a simplified tri-trophic legume-symbiont-PGPR system consisting of $M$. truncatula A17 Jemalong, its symbiotic mutualist S. meliloti strain Rm8530, and the PGPR B. subtilis 
strain UD1022 (Glazebrook and Walker, 1989; Bishnoi et al., 2015; Rosier, 2016). The organisms in this model were specifically selected to be representative due to their comprehensively described genetics and lifestyles. The primary goal of this work is to identify interspecies interactions between PGPR, which may influence their plant beneficial activities in the legumeRhizobia symbiosis. By using the tri-trophic model as a platform for testing phenotypic outcomes of the "consortium," more fundamental questions regarding the interspecies interactions can be developed. Specifically, does the PGPR and legume symbiont consortia act synergistically to increase $M$. truncatula plant growth, how do the different PGPR species directly or indirectly interact with one another, and do those interactions influence their ability to interact with and confer benefits to the plant? We employed phenotypic and molecular assays to evaluate the legume-Rhizobia-PGPR interactions.

\section{MATERIALS AND METHODS}

\section{Bacterial Growth}

Primary cultures of all bacteria strains were grown and maintained on TYC media [TY media (Beringer, 1974) liquid or agar supplemented with $1 \mathrm{mM} \mathrm{CaCl} 2$ ] with appropriate antibiotics. Subcultures of S. meliloti strain Rm8530 and Bacillus subtilis strain UD1022 (hereafter "UD1022") prepared for biofilm treatments were sub-cultured into minimal glutamate mannitol (MGM) and low phosphate $(0.1 \mathrm{mM})$, as described in Marketon and Gonzalez (2002). Both UD1022 and Rm8530 strains were grown at $30^{\circ} \mathrm{C}$ for all experiments. AT medium for culturing pre-induced A. tumefaciens KYC55 was prepared as described in Joelsson and Zhu (2005). Strains used in this work are listed in Table 1.

\section{Plant Growth and Co-inoculation}

Seeds of M. truncatula A17 cv Jemalong were acid scarified for $6 \mathrm{~min}$ and sterilized with $3 \%$ bleach for $3 \mathrm{~min}$. Seeds were imbibed in sterile water at $4^{\circ} \mathrm{C}$ overnight, rinsed and placed in sterile petri dish, and germinated covered overnight at room temperature (Garcia et al., 2006). Germinated seeds were placed in sterile Magenta ${ }^{\circledR}$ (Magenta Corp.) jars with Lullien’s solution (Lullien et al., 1987), sealed with $3 \mathrm{M}^{\mathrm{TM}}$ MicroPore ${ }^{\mathrm{TM}}$ surgical tape, and grown in a controlled environmental chamber at $55 \%$ relative humidity and a $14 \mathrm{~h}, 22^{\circ} \mathrm{C}$ day $/ 10 \mathrm{~h}, 18^{\circ} \mathrm{C}$ night cycle. After 6 days of growth, plants were inoculated with bacteria treatments, with 10 plants per treatment. $\mathrm{Rm} 8530$ was grown to $\mathrm{OD}_{600}=0.8$ and UD1022 was grown at $\mathrm{OD}_{600}=1.0$. Bacteria were spun down, washed three times in sterile $\mathrm{H}_{2} \mathrm{O}$, and resuspended with $0.5 \times$ Lullein's solution with $\mathrm{Rm} 8530$ final $\mathrm{OD}_{600}=$ of 0.02 and UD1022 $\mathrm{OD}_{600}=0.01$ (in Magenta jar). Plants were harvested 7 weeks after inoculation. Experiment was repeated three times.

\section{Cross-Streak for Growth Inhibition Analysis}

Rm8530 bacteria were grown to $\mathrm{OD}_{600}=0.8$ and UD1022 $\mathrm{OD}_{600}=1.5$. Both cultures were diluted to $\mathrm{OD}_{600}=0.5$ with sterile $\mathrm{H}_{2} \mathrm{O}$. Bacteria were streaked on TYC agar plates using a sterile loop in a cross pattern.

\section{Biofilm Assays \\ Preparation of Cell-Free Supernatant (CFS) Derived From UD1022 for Biofilm Assays}

UD1022 was inoculated from a single plate colony into $5 \mathrm{ml}$ of TYC and grown overnight (16 h) and then diluted 1:50 in $50 \mathrm{ml}$ of MGM in a sterile $150 \mathrm{ml}$ flask and grown shaking for $8 \mathrm{~h}$ to an $\mathrm{OD}_{600}=0.8-1.0$. Cultures were centrifuged $10 \mathrm{~min}$, $4^{\circ} \mathrm{C}$ at 4,000 RPM. Culture supernatant was filter-sterilized with $0.22 \mu \mathrm{m}$ membrane (Steriflip ${ }^{\circledR}$, EMD Millipore) under gentle vacuum. Supernatant was centrifuged and filter-sterilized once more. A sub-fraction was heat treated in water bath overnight at $65^{\circ} \mathrm{C}$.

\section{Preparation of Biofilm Treatments}

Biofilm assays were based on methods found in O'Toole et al. (1999) and Rinaudi and Gonzalez (2009). Rm8530 was grown $48 \mathrm{~h}$ in TYC to $\mathrm{OD}_{600}=1.5-2.0$, and then cells were "preconditioned" by diluting 1:100 to MGM media and grown shaking $48 \mathrm{~h}$ to $\mathrm{OD}_{600}=0.8$. Stocks of treatments were made by centrifuging and re-suspending cell pellets with fresh MGM, or UD1022 CFS, UD1022 "heat-treated" CFS to a total of $5 \%$ by volume in MGM. One hundred microliters of these treatment stocks was then aliquoted to 96-well plates with eight replicate wells per treatment. Plates were sealed with Parafilm ${ }^{\circledast}$ (Bemis Company, Inc.) and placed in a shaker at $30^{\circ} \mathrm{C}$ and measured at 24, 48, and $72 \mathrm{~h}$. Experiment was repeated three separate times.

Plates were then emptied and gently rinsed three times with sterile water, dried, and stained $20 \mathrm{~min}$ with $150 \mu \mathrm{l}$ of $0.1 \%$ crystal violet. Plates were emptied, rinsed gently three times with sterile water. Crystal violet (CV) was solubilized with modified biofilm dissolving solution (MBDS) (Tram et al., 2013). OD 570 of CV was then measured using Wallac 1420 Plate Reader (PerkinElmer Life and Analytical Science, Wallac Oy, P.O. Box 10, FIN-20101 Tuku, Finland).

\section{Gene Expression Reporter Assays}

Reporter lines for Rm8530 were provided by Dr. Max Teplitski of the University of Florida. All cultures grown in liquid TYC broth shaking at $225 \mathrm{RPM}$ at $30^{\circ} \mathrm{C}$. Bacteria primary cultures were grown with appropriate antibiotics $48 \mathrm{~h}$ to $\mathrm{OD}_{600}=2.0$ 3.0. Cells were further prepared as described in the Biofilm Assays section and 29 replicate wells were included per treatment. Every $24 \mathrm{~h}$, total well fluorescence and cell growth were measured using Wallac 1420 Plate Reader (PerkinElmer Life and Analytical Science, Wallac Oy, P.O. Box 10, FIN-20101 Tuku, Finland). Data were reported as fluorescence counts/ $\mathrm{OD}_{570}$ (Gao et al., 2012). After the $72 \mathrm{~h}$ measurement, 96-well plates were processed as described in the Biofilm Assays section above to assess qualitative biofilm formation. Gene reporter assays were repeated three times.

\section{Statistical Analysis}

For plant growth biological data and biofilm analysis, data normality and homogeneity were reviewed prior to analysis of variance (ANOVA). No data transformations were required. One-way ANOVA was used to test for differences between treatments. When $F$ ratios were significant $(p<0.05)$, treatment 
TABLE 1 | Bacterial strains used in this study.

\begin{tabular}{|c|c|c|}
\hline Strain & Genotype & References or Sources \\
\hline Rm1021 & SU47 str-21 expR102::/SRm2011-1 & Meade et al., 1982 \\
\hline Rm8530 & Sinorhizobium meliloti Rm1021 exp $R^{+}$ & Glazebrook and Walker, 1989 \\
\hline Rm8530 Sinl-gfp & with integrated pMG309 & Gao et al., 2012 \\
\hline Rm8530 WggR-gfp & with integrated pMG310 & Gao et al., 2012 \\
\hline \multirow[t]{2}{*}{ KYC55 } & Agrobacterium tumefaciens & Zhu et al., 2003 \\
\hline & (pJZ410) (pJZ384) (pJZ372) & \\
\hline UD1022 & Bacillus subtilis & Bishnoi et al., 2015 \\
\hline UD1022 ytnP- & UD1022ytnP::erm & Dr. Pascale Beauregard \\
\hline
\end{tabular}

TABLE 2 | Primer sequences used in this study.

\begin{tabular}{|c|c|c|c|}
\hline Primer & Sequence $5^{\prime}-3^{\prime}$ & Amplicon & Source length \\
\hline Rm8530 rpoE1-fw & CGAGGAAGAGGTCCTGGAAT & $100 \mathrm{bp}$ & Trabelsi et al., 2009 \\
\hline Rm8530 rpoE1-rv & GACGCAGTCCTGCAACAGAT & & \\
\hline Rm8530 Sinl F & CCGGAAATCCGTAGTGCGTC & $76 \mathrm{bp}$ & Gurich and González, 2009 \\
\hline Rm8530 Sinl R & ATGCGCGATCCTGGGAGATT & & \\
\hline Rm8530 WggR F & TCCGTTCGCAGACTTGGAG & $107 \mathrm{bp}$ & This work \\
\hline Rm8530 WggR R & CGAGCGAATCATCTCCGTCA & & \\
\hline
\end{tabular}

means were compared via Tukey Kramer HSD using SAS-JMP (Cary, NC, United States).

For gene expression reporter results analysis, ANOVA was used to test for treatment differences. Where $F$-ratios were significant $(p<0.05)$, treatment means were compared via Tukey-Kramer test (JMP, SAS Institute Inc, 1989-2019). Non-parametric analyses (Kruskal-Wallis test) were utilized if data failed to meet parametric assumptions. Where $H$-values (Kruskal-Wallis test statistic) were significant $(p<0.05)$, treatment means were compared via Kruskal-Wallis multiple comparison $Z$-value test using NCSS software (Hintze, 2000).

\section{Gene Expression Analysis Using Semi-Quantitative Reverse Transcription PCR (qRT-PCR)}

\section{Primer Design for qRT-PCR}

Gene sequences were derived from GenBank; S. meliloti 1021 sequences were derived from genome (accession: AL591688.1) and mega-plasmids pSymA (accession: AE006469.1). The sinI primer pair from Gurich and González (2009) and the rpoE1 primer pair from Trabelsi et al. (2009). Primers from this work were designed using GenScript Real-time PCR (TaqMan) Primer Design ${ }^{1}$. Amplicon size was restricted to $150 \mathrm{bp}$ or less. All primer sequences (Table 2) were cross-checked on all strain sequences to ensure species specificity.

\section{Experimental Protocol for qRT-PCR}

For qRT-PCR analysis, cells were "pre-conditioned" on MGM media as described under the Biofilm Assays section. Cells were pelleted and re-suspended in fresh MGM plus the treatment. Co-inoculations were combined as $\mathrm{Rm} 8530 \mathrm{OD}_{600}=0.8$ and UD1022 $\mathrm{OD}_{600}=0.2$. Luteolin treatments contained a final

${ }^{1}$ https://www.genscript.com/ssl-bin/app/primer concentration of $5 \mu \mathrm{M}$ luteolin. Treatments were grown shaking at $30^{\circ} \mathrm{C}$, and $1.5 \mathrm{ml}$ samples were collected at time points of 12 and $24 \mathrm{~h}$, centrifuged, decanted, and flash frozen in liquid nitrogen. RNA was isolated using NucleoSpin ${ }^{\circledR}$ RNA from Macherey-Nagel (Düren, Germany). cDNA was generated with 500 ng of RNA using High Capacity cDNA Reverse Transcription Kit from Applied Biosystems ${ }^{2}$ and qPCR was performed using PerfeCTa $^{\circledast}$ SYBR $^{\circledast}$ Green SuperMix, ROX, Quanta Biosciences (Gaithersburg, MD), and run on Eppendorf Mastercycler ${ }^{\circledR 3} \mathrm{ep}$ realplex ${ }^{2}$. Experiments were repeated three times.

\section{Expression Analysis of qRT-PCR}

The relative change in gene expression was calculated with the $2^{-\Delta \Delta C t}$ method as described in Schmittgen and Livak (2008), which calculates the expression of the gene of interest relative to the internal control in the treated sample compared with the untreated control. The internal control gene for Rm8530 is rpoE1. Genes were considered to be differentially expressed if the fold change in expression was $\geq 2$ or $\leq-2$.

\section{AHL Biosensor Assays for QQ Analysis}

Preparation of the AHL biosensor Agrobacterium tumefaciens KYC55 was as described in Joelsson and Zhu (2005) with modifications. KYC55 pre-induced cells were inoculated 1:1,000 into MGM medium for X-Gal soft agar 6-well plates. Preinduced KYC55 cells were made as described in Joelsson and Zhu (2005). Soft agar plates were treated the same day they were poured. UD1022 was inoculated from a fresh plate streaked from glycerol stock into TYC and grown shaking $30^{\circ} \mathrm{C}$ for $5 \mathrm{~h}$ to $\mathrm{OD}_{600}=1.5$, then sub-cultured $1: 100$ to MGM media and grown shaking $30^{\circ} \mathrm{C}$ for $20 \mathrm{~h}$ to $\mathrm{OD}_{600}=0.5$. Treatments were made using these cultures mixed into sterile

\footnotetext{
${ }^{2}$ www.appliedbiosystems.com

${ }^{3}$ www.eppendorf.com
} 
micro-centrifuge tubes with standard C8-AHL and 3-oxo-C16AHL to a final concentration of $10 \mu \mathrm{M}$ in a volume of $200 \mu \mathrm{l}$. Controls contained standard AHL only. Treatments were incubated shaking $30^{\circ} \mathrm{C}$ for $24 \mathrm{~h}$. Samples were then centrifuged at $16,000 \times g$ for $10 \mathrm{~min}$ at $4^{\circ} \mathrm{C}$. Supernatants were transferred to new sterile tubes and sterilized open in a biosafety cabinet under UV light for $30 \mathrm{~min}$. Two microliters of treatments was applied to KYC55 X-Gal soft agar sixwell plates and allowed to dry. Two treatment replicates were included on two separate six-well plates. AHL biosensor assay was repeated twice.

\section{Sequence Homology and Alignment}

The FASTA protein sequence YtnP protein in B. subtilis subsp. subtilis str. 168 (sequence NP_390867.1) was queried using tblastn search translated nucleotide databases using a protein query for B. subtilis UD1022 nucleotide reference sequence (NZ_CP011534.1). The protein sequence for UD10222 YtnP has 281 amino acids and has a molecular weight of $31.8 \mathrm{kDa}$. The alignment of UD1022 YtnP, AiiA, and other MBL sequences was performed in MEGA (Tamura et al., 2011) by using the software MUSCLE (Edgar, 2004).

\section{Construction of YtnP Mutant}

The $y \operatorname{tn} P$ gene disruption $B$. subtilis subsp. subtilis trpC2 ytnP::erm (Koo et al., 2017) was obtained from the Bacillus Genetic Stock Center and transferred into B. subtilis UD1022 by SPP1 phage transduction (Yasbin and Young, 1974).

\section{YtnP Protein Expression and Purification}

The B. subtilis UD1022 ytnP specific sequence was submitted to University of North Carolina School of Medicine Center for Structural Biology (NIH grant P30CA016086) for protein expression and purification. Workers sent the sequence to GenScript for gene synthesis and subcloning into a pET expression vector that contains an $\mathrm{N}$-terminal His tag followed by a TEV site for tag removal during purification [pET-28a(+)TEV]. The $y t n P:: E$. coli construct expression was done at UNC using their autoinduction expression system. Purification was performed using a Ni-affinity step, TEV protease tag removal, subtractive Ni-affinity step to separate out the tag, and size-exclusion chromatography to remove potential protein contaminants.

\section{RESULTS}

\section{Co-inoculation of UD1022 and Rm8530 Do Not Synergistically Promote Plant Growth}

M. truncatula plants were co-inoculated with B. subtilis UD1022 and S. meliloti Rm8530 6 days after germination and analyzed 7 weeks after inoculation for biomass and nodulation. Though the co-inoculation of Rm8530 and UD1022 resulted in no statistical difference in shoot biomass (Figure 1A, $p=0.06$ ), there was a slight decrease in observable shoot growth (Figure 1C). There was no statistical difference in nodule numbers (Figure 1B, $p=0.59$ ) between co-inoculated plants from those inoculated with Rm8530 alone. These results indicate that the addition of the PGPR UD1022 to the symbiotic strain Rm8530 did not increase plant health, contrary to results from similar studies (Fox et al., 2011; Morel et al., 2015). We speculated that the lack of growth promotion by the co-inoculation may be due to the antagonistic activity of UD1022 against Rm8530. A standard cross-streak compatibility assay on solid media determined no direct growth inhibitory effect between the two bacteria (Supplementary Figure S1).

\section{UD1022 Interacts Indirectly With Rm8530 by Interfering With Rm8530 Biofilm and QS}

UD1022 had no observable direct effects on Rm8530 growth; consequently, treatments of UD1022 culture filtrate supernatant (CFS) were tested for indirect influence on the $\mathrm{Rm} 8530$ functional phenotype of biofilm production. Biofilm formation by the symbiotic Rm8530 strain is required for efficient nodulation (González et al., 1996) and was evaluated by the semiquantitative O'Toole assays (O'Toole et al., 2000) in treatments with UD1022 CFS. Biofilm of Rm8530 cultured with 5\% by volume of UD1022 CFS was significantly reduced from that of control (Figure 2, $p<0.0001$ ). Growth of Rm8530 with heattreated CFS treatment resulted in restoration of control quantities of biofilm (Figure 2, $p=0.86$ ), suggesting that the active factor of UD1022 CFS may be a heat-unstable molecule such as a protein.

\section{UD1022 Affects Rm8530 QS-Controlled Biofilm Gene Expression}

The relative expression of two key Rm8530 QS genes were measured in response to co-culture with UD1022 CFS and in co-culture with live UD1022 cells using qRT-PCR. Rm8530 sinI relative gene expression increased by 4 -fold and $w g g R$ relative gene expression decreased by nearly threefold in treatments grown with UD1022 (Figure 3). These UD1022 live-cell coculture qRT-PCR results reflected the same trend of expression as observed in the GFP gene expression reporter assays treated with UD1022 CFS (Figure 4): upregulation of sinI and downregulation of $w g g R$. Treatments with the M. truncatulaspecific flavonoid luteolin (Peters et al., 1986) were included in the qRT-PCR expression analysis to evaluate possible plant host role in the interaction of the bacteria. Luteolin induces nod gene expression in S. meliloti, an important initial signaling mechanism to initiate legume-bacteria symbiosis. Rm8530 QS gene expression, as expected was not directly affected by the presence of luteolin alone. However, the presence of luteolin in Rm8530-UD1022 co-culture significantly enhanced the gene expression changes observed in bacteria co-cultures. The increase in $\sin I$ relative expression doubled to nearly 8 -fold and $w g g R$ decreased expression was extended to 3.4-fold (Figure 3). This could indicate that, in the rhizosphere, plant signaling factors such as flavonoids may exacerbate the PGPR interactions causing the changes in QS gene expression. 


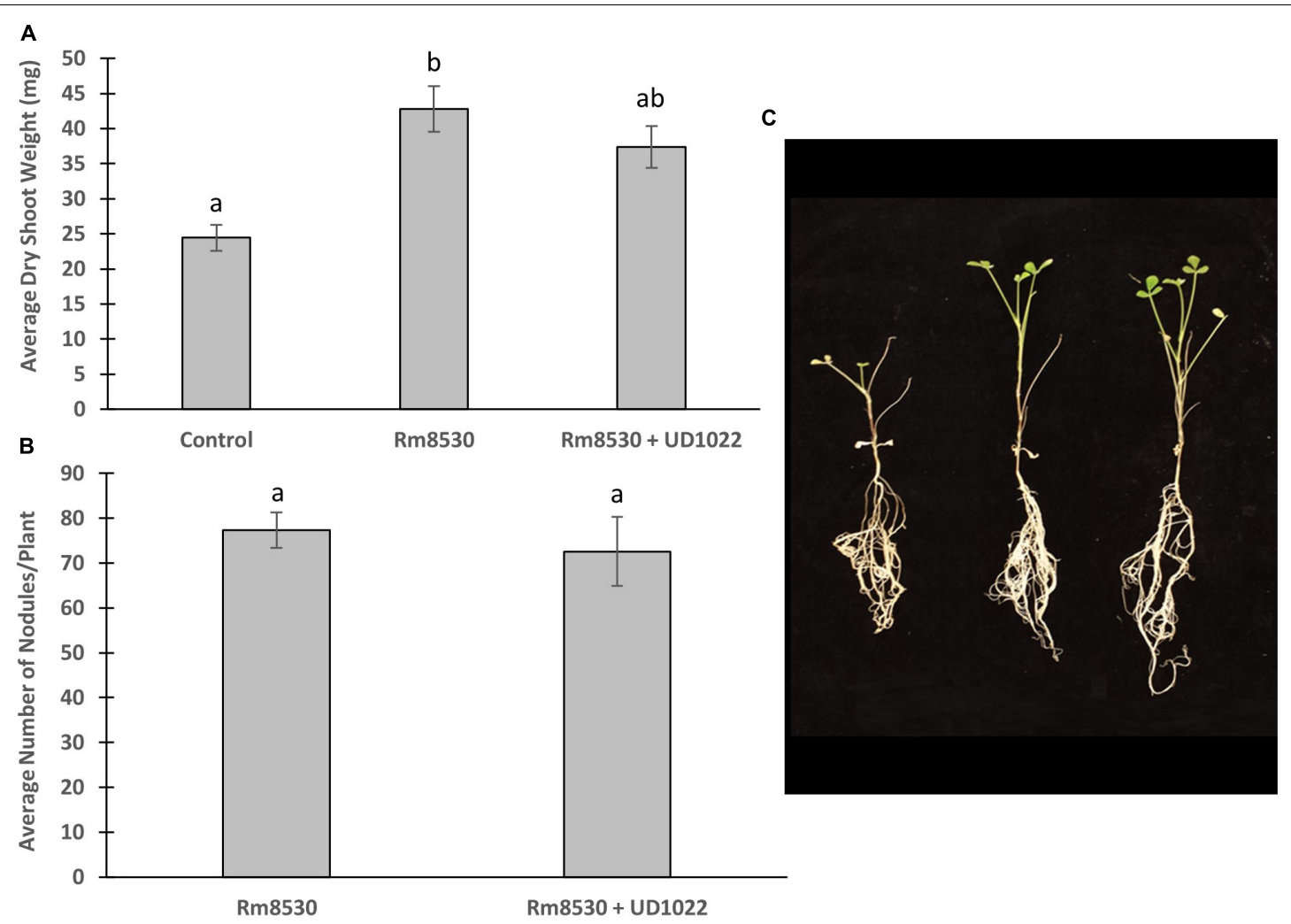

FIGURE 1 | Co-inoculated plant growth and nodulation. (A) Average plant dry weight of Rm8530 treated control plants and co-inoculated Rm8530 \& UD1022 plants did not differ statistically ( $p$-value of 0.06 ). (B) There was no statistical difference between average counts of nodules between treatments ( $D$-value of 0.59$)$. (C) Overall plant growth of both treatments was greater than control (first plant), but no differences were observed between Rm8530 treatment (second plant) and Rm8530 \& UD1022 co-inoculation (third plant).

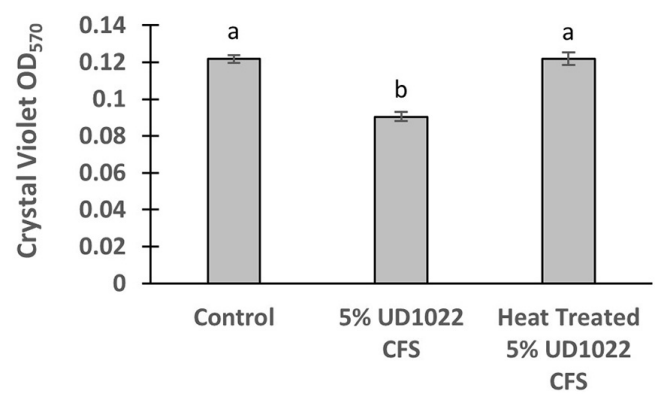

FIGURE 2 | Rm8530 biofilm formation assay. Treatment with 5\% UD1022 CFS significantly reduced the formation of biofilm by Rm8530 ( $p$-value of $<0.0001)$. Treatment with 'heat treated' UD1022 CFS showed no significant difference compared to the control ( $p$-value of 0.86 ).

\section{UD1022 Is Positive for QQ Activity Against Rm8530}

The Rm8530 QS gene expression response patterns coupled with the restoration of WT Rm8530 biofilm formation in UD1022 heat treated CFS treatments suggest that UD1022 may be affecting Rm8530 QS through enzymatic activity of a protein. Interference of QS through interspecific enzymes is termed quorum quenching and can be enacted at several levels of QS regulation, including targeting signal biosynthesis, signal receptors, and direct cleavage of QS signal molecules, including AHLs (Fetzner, 2015). QQ enzymes have been characterized in many soil bacteria including Agrobacterium and Bacillus genera (Chan et al., 2016).

QQ activity of UD1022 was assessed using the bioreporter strain Agrobacterium tumefaciens KYC55 to detect a wide range of AHLs and reports through $\beta$-galactosidase activity (Zhu et al., 2003). UD1022 cultures were incubated with $10 \mu \mathrm{M}$ purified $\mathrm{N}$-octanoyl-L-homoserine lactone (C8-AHL) or N-3-oxo-hexadecanoyl-L-homoserine lactone (3-oxo-C16AHL) (Caymen Chemicals) for $24 \mathrm{~h}$ on KYC55 X-Gal plates. Treatments of UD122 with 3-oxo-C16-AHL showed significant reduction in detectable AHL signal as compared to AHL only (Figures 5A,B), while C8-AHL showed no discernable difference from the control (Supplementary Figure S2B). Thus, UD1022 displays QQ activity, which appears to be geared toward longchain AHLs.

\section{UD1022 QQ Through the Lactonase YtnP Protein}

Several classes of bacterial enzymes QQ through inactivating AHLs, including lactonases and acylases (Chan et al., 2016). 


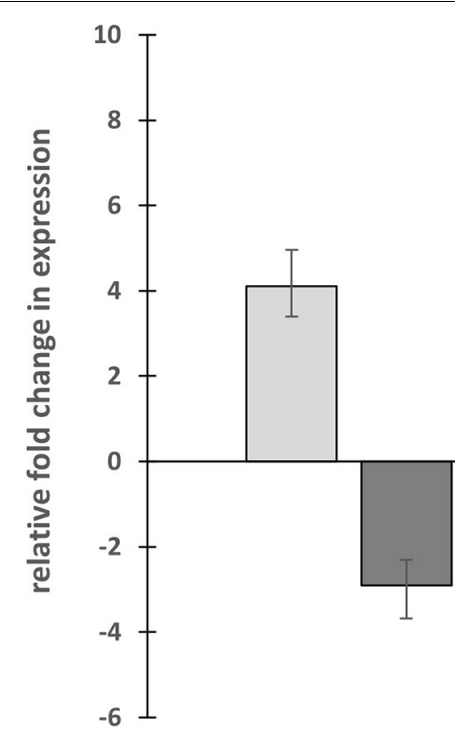

Co-culture with UD1022

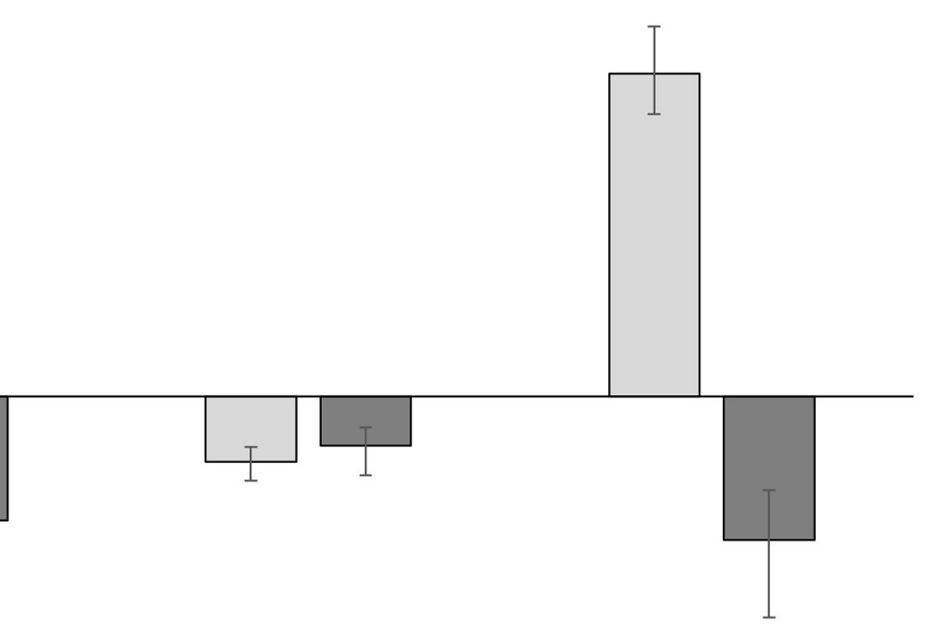

Luteolin
UD1022 \& Luteolin

$\square \operatorname{sinl} \square w g g R$

FIGURE 3 | Relative fold changes in expression of Rm8530 sinl and wggR in co-culture with UD1022. Co-culture with UD1022 increased the relative expression of Rm8530 sin/ by 4-fold. The presence of luteolin (which represents the condition of Rm8530 upregulating nod genes) doubled the effect of UD1022 on Rm8530 sinl, increasing expression to nearly 8-fold. Luteolin alone did not meet the threshold of 2-fold change in Rm8530 sin/ expression. Co-culture with UD1022 decreased the relative expression of Rm8530 wggR by 3-fold. The presence of luteolin slightly enhanced the effect of UD1022 on Rm8530 wggR, increasing to 3.4-fold. Luteolin alone did not change Rm8530 wggR expression. Standard error bars reflect the range of the relative fold change in gene expression in response to the treatment.

A search of the literature for lactonases specifically identified in B. subtilis species yielded the putative lactonase YtnP protein in B. subtilis NCIB3610 (Schneider et al., 2012). The alignment of YtnP protein sequence with UD1022 returned a 98.44\% identity. Using MUSCLE (Edgar, 2004) alignments of the reference protein B. subtilis strain $168 \mathrm{YtnP}$ (NP_390867.1) and UD1022 YtnP sequences revealed the hallmark metallohydrolase $\mathrm{HXHXDH}$ and HXXGH metal binding motifs as well as a phosphorylated Ser36 residue. To determine if the UD1022 YtnP lactonase protein contributes to the QQ patterns observed, we introduced a $y \operatorname{tnP}$ deletion cassette in UD1022 ("UD1022 $y t n P^{-”)}$ ). In AHL co-incubation assays, UD1022 ytn $P^{-}$treatments with 3-oxoC16-AHL showed that AHL degradation was less extensive than that of UD1022 WT (Figure 5C). It is likely that there are additional QQ active proteins produced by UD1022. Indeed, up to six other probable MBL-like fold sequences having the HXHXDH motif have been identified in UD1022 (data not shown).

The UD1022 specific $y \operatorname{tn} P$ sequence was submitted to the University of North Carolina School of Medicine Center for Structural Biology (NIH grant P30CA016086) for protein expression and purification. Purified YtnP protein was applied at three different concentrations to $10 \mu \mathrm{M}$ concentrations of 3-oxo-C16-AHL and C8-AHL. The biosensor reporter showed no degradation of 3-oxo-C16-AHL with treatment of $5 \mu \mathrm{g} / \mathrm{ml}$ YtnP (Figure 5D). Long-chain AHL degradation comparable to UD1022 WT live cell treatments was observed with 50 $\mu \mathrm{g} / \mathrm{ml}$ YtnP incubation (Figure 5E). Treatments of $500 \mu \mathrm{g} / \mathrm{ml}$
YtnP completely abolished detectable levels of 3-oxo C16AHL (Figure 5F). Incubation of UD1022 YtnP with C8-AHL, interestingly, resulted in degradation of the short-chain AHL at 50 and $500 \mu \mathrm{g} / \mathrm{ml}$ (Supplementary Figures S2E,F, respectively). This demonstrates unequivocally that UD1022 QQ activity is carried out through the YtnP lactonase protein.

\section{DISCUSSION}

Understanding interactions of PGPR in consortia is critical for predicting rhizo-microbiome function in the environment and in agroecosystems. This is especially relevant as biologicalbased crop solutions become more widely marketed and adopted. Several examples of PGPR co-inoculations using $S$. meliloti resulting in significant improvements of Medicago spp. plant growth have been reported. Co-inoculation of Delftia spp. JD2, a diazotrophic, IAA-producing PGPR, with $S$. meliloti U143 onto $M$. sativa increased nodulation (Morel et al., 2011) and increased shoot and root dry weights by 13 and 34\%, respectively (Morel et al., 2015). Fox et al. (2011) found dual inoculation of S. meliloti WSM419 and the PGPR Pseudomonas fluroescens WSM3457 onto M. truncatula enhanced nodule initiation rates, resulting in increased number of crown nodules and more overall $\mathrm{N}$ accumulation. S. meliloti B399, a commercial alfalfa inoculant closely related to strain Sm1021, co-inoculated with Pseudomonas spp. FM7d nearly doubled shoot dry weight and increased nodule number on 


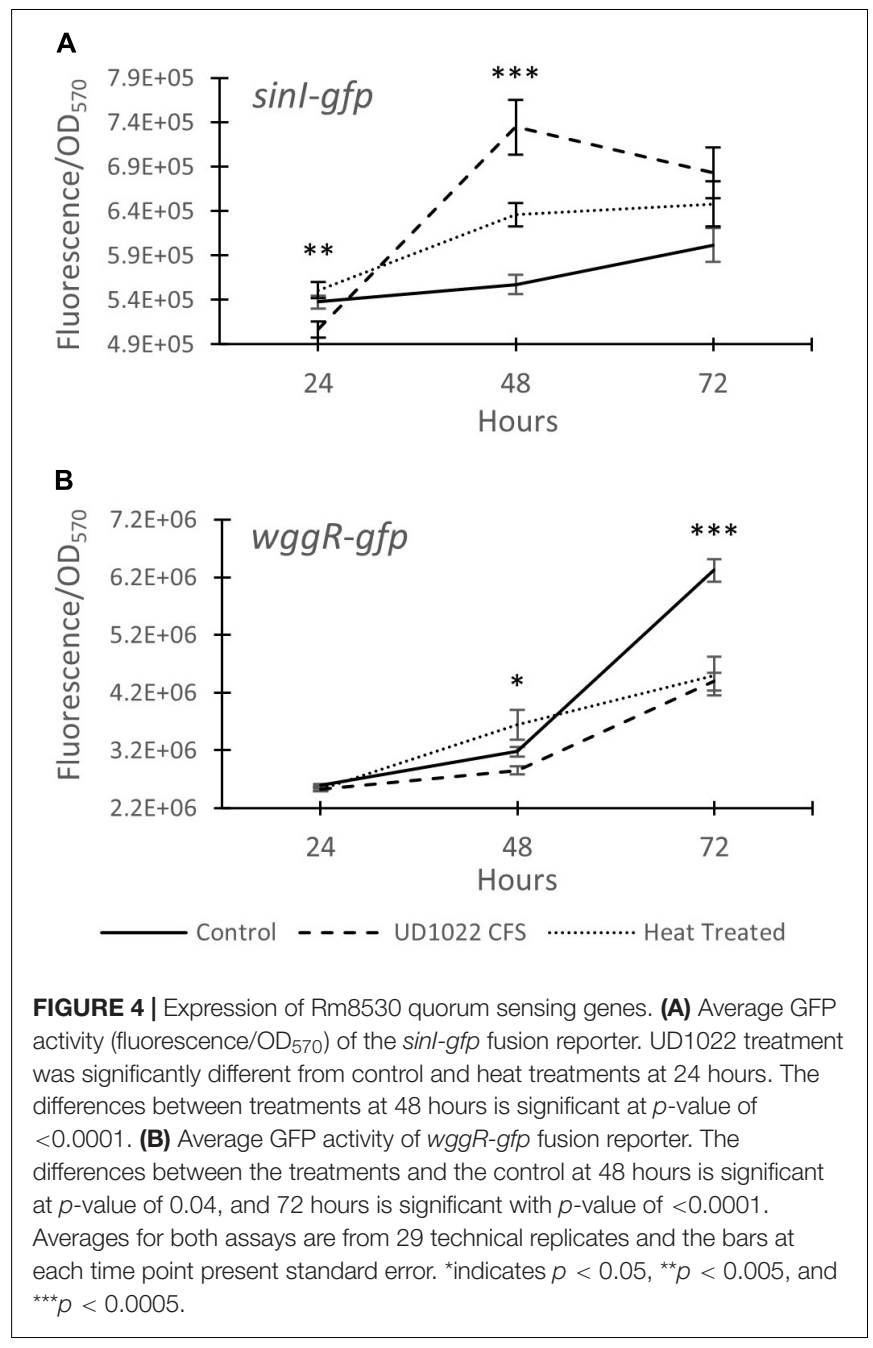

M. sativa L. cv Bárbara SP (INTA Manfredi). Though coinoculation of B399 with Bacillus spp. M7c had significantly higher shoot dry weight, it did not increase nodule number (Guiñazú et al., 2010).

However, not every instance of PGPR dual inoculation with S. meliloti has been reported to be beneficial. The dual inoculation of the PGPR B. simplex $30 \mathrm{~N}-5$ with S. meliloti 1021 onto $M$. truncatula resulted in no significant difference in shoot height, plant dry weight or nodule number over that of S. meliloti 1021 control (Maymon et al., 2015). This contrasted with their previous work, which showed beneficial growth effects of $B$. simplex $30 \mathrm{~N}-5$ when co-inoculated with Rhizobium leguminosarum bv. viciae $128 \mathrm{C} 53$ onto pea (Pisum sativum) (Schwartz et al., 2013). Our study using the $\exp R+$ S. meliloti strain Rm8530 co-inoculated with B. subtilis UD1022 also resulted in no significant enhancement of plant growth or nodule number. While other work has yet to query the mechanisms of bacterial interaction, which may account for the non-synergistic plant effects of these rhizobia-PGPR coinoculations, this work reveals a potential, indirect mechanism of bacterial interaction.
Biofilm formation is important in soil and root-associated bacteria for motility and exchange of signals and metabolites (Angus and Hirsch, 2013; Bogino et al., 2013; Amaya-Gómez et al., 2015). S. meliloti biofilms have been shown to play a critical role in motility toward and initiation of nodulation with the Medicago spp. plant root (González et al., 1996; Pellock et al., 2000; Hoang et al., 2008). Here, we used Rm8530 biofilm formation as a functional reporter for negative activity by UD1022 and found clear evidence of UD1022 inhibition of Rm8530 biofilm formation. S. meliloti biofilm formation is dependent on an intact ExpR/SinI QS system, which is well described for both strains Rm1021 and its ExpR+ relative Rm8530. Importantly, the Rm8530 QS system has been shown to regulate a key symbiotically active component of their biofilms, the low-molecular-weight galactoglucans referred to as EPS II (Rinaudi and Gonzalez, 2009).

Based on the negative effect of UD1022 on Rm8530 biofilm, we hypothesized that UD1022 may be interfering with the QS-controlled molecular regulation of biofilm production. The Rm8530 QS genes $\sin I$ and $w g g R$ were selected to test the effect of UD1022 on the QS pathway, including upstream QS signal molecule synthesis $(\sin I)$ and downstream EPS II polymerization $(w g g R)$. Using UD1022 CFS treatments on Rm8530- $g f p$ expression reporters and subsequent validation with qRT-PCR of live-cell co-cultures, we found that UD1022 significantly activated $\sin I$ transcription and reduced $w g g R$ transcription. McIntosh et al. (2009) described that sinIpromoter activation occurs at nearly 10 -fold lower levels of AHLs than required for its downregulation. WggR activation requires the presence of the transcriptional regulator ExpR and the SinIspecific AHLs $C_{16: 1}$-AHL and oxo- $C_{16: 1}$-AHL (McIntosh et al., 2009; Gao et al., 2012). The wggR-gpf reporter in $\mathrm{Rm} 8530$ sinI background was more sensitive to $C_{16: 1}$-AHL than 3-oxo- $C_{16: 1^{-}}$ AHL. Expression of $w g g R$ increased in a dose-dependent manner with close to WT levels at 40-1500 nM C 16:1-AHL and 200-1,500 nM oxo-C $16: 1$-AHL (Gao et al., 2012). Consequently, UD1022 treatment appeared to mimic an expression pattern of the QS genes similar to their response to low AHL signal molecule concentration conditions.

The regulatory network of S. meliloti ExpR/SinI is intricately controlled through AHL acyl chain length, acyl chain substitutions, and concentration of AHL molecules (Bartels et al., 2007; Calatrava-Morales et al., 2018). Gene expression for sinI synthase is positively regulated by low concentrations of AHLs (1-40 nM) and negatively regulated by high concentrations of AHLs (>40 nM), allowing the ExpR transcriptional regulator of $\sin I$ to be sensitive to the AHL substrate it is responsible for producing (Baumgardt et al., 2014). The expression of $w g g R$ is oppositely regulated, requiring upwards of $150 \mathrm{nM} \mathrm{C} 16: 1$-AHL for increased $w g g R$ GFP expression reporter activity (Gao et al., 2012). Lower concentration of AHLs in UD1022 treatments would support the patterns of increased $\sin I$ and decreased $w g g R$ expression (schematic in Figure 6). QQ activities could be promising as a prospective tool to improve plant health and bypass antibiotic resistance in the development of biological products combating plant pathogens (Grandclément et al., 2016; Rodríguez et al., 2020). Many screening techniques have 


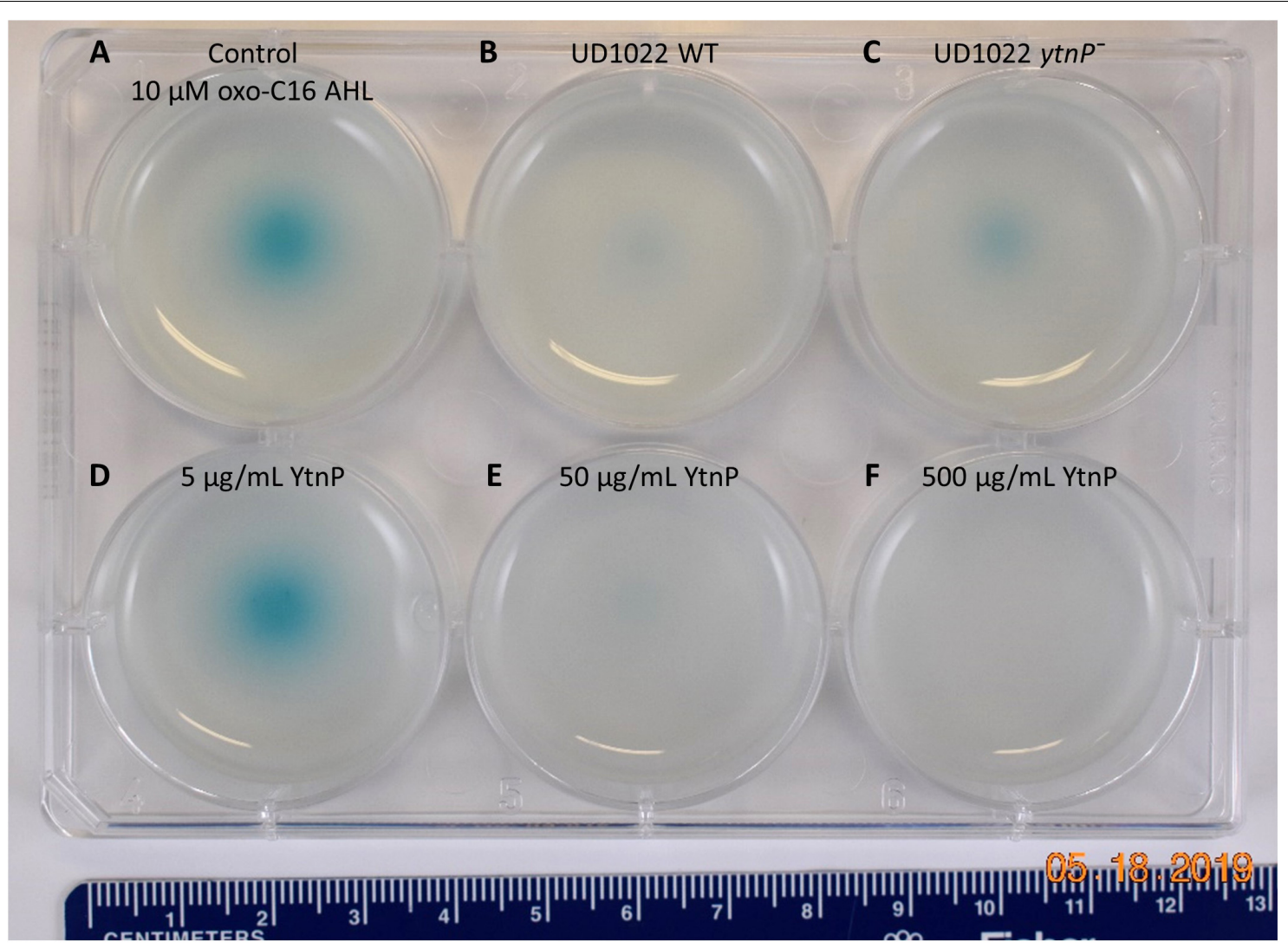

FIGURE 5 | UD1022 quorum quenching biosensor assay plate. The biosensor KYC55-X-gal soft agar plate treated with UD1022-AHL co-cultures. From top left across (A) control treatments of standard AHLs with no UD1022. (B) QQ activity of UD1022 culture with 3-oxo-C16-AHL (C) UD1022 ytnP- mutant cultured with AHL (D) $5 \mu \mathrm{g} / \mathrm{mL}$ pure UD1022 YtnP protein incubated with AHL, (E) $50 \mu \mathrm{g} / \mathrm{mL}$ YtnP protein, (F) $500 \mu \mathrm{g} / \mathrm{mL}$ YtnP protein. *Brightness of image increased by $20 \%$, which did not increase pigment intensity or saturation.

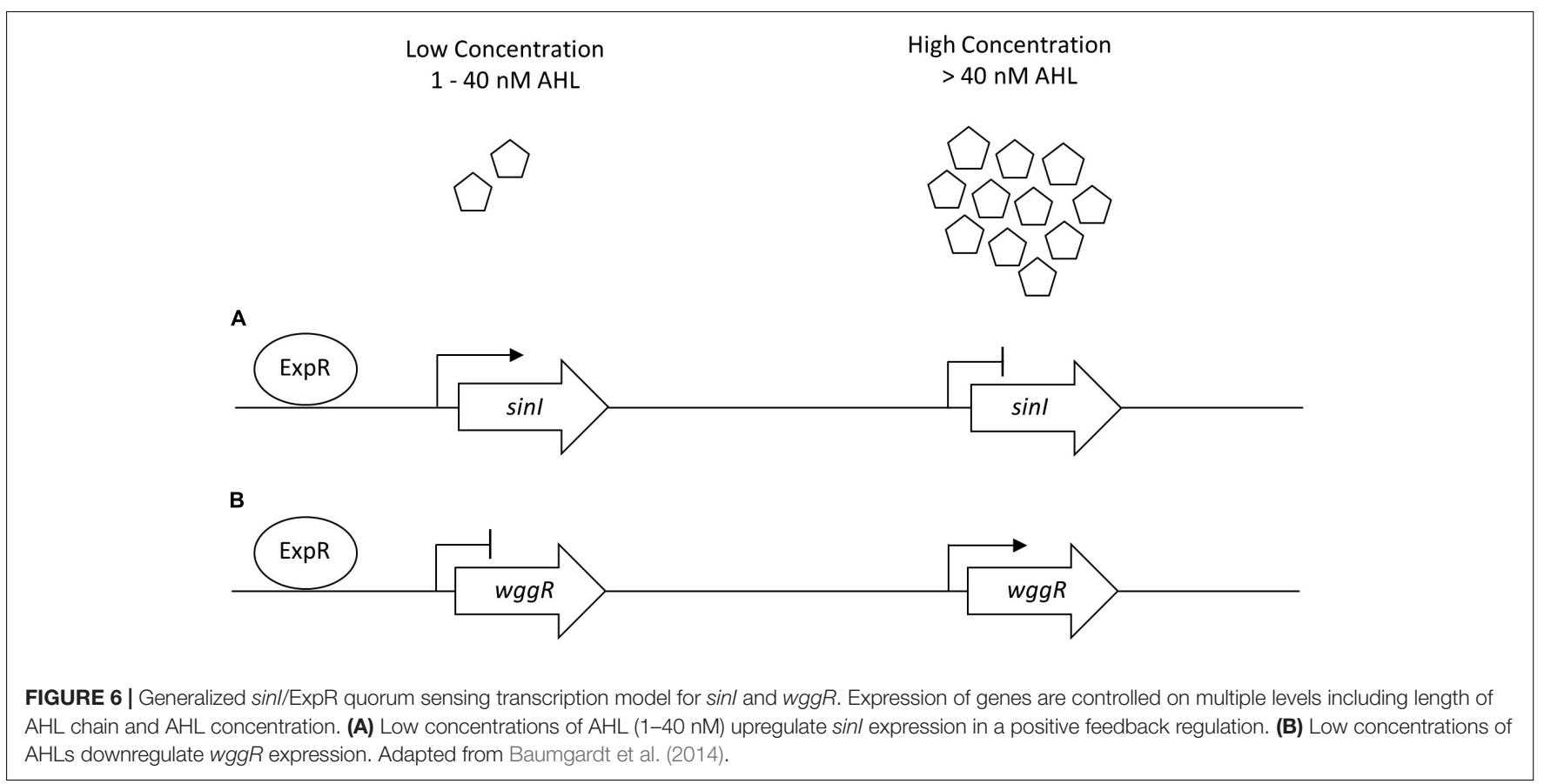




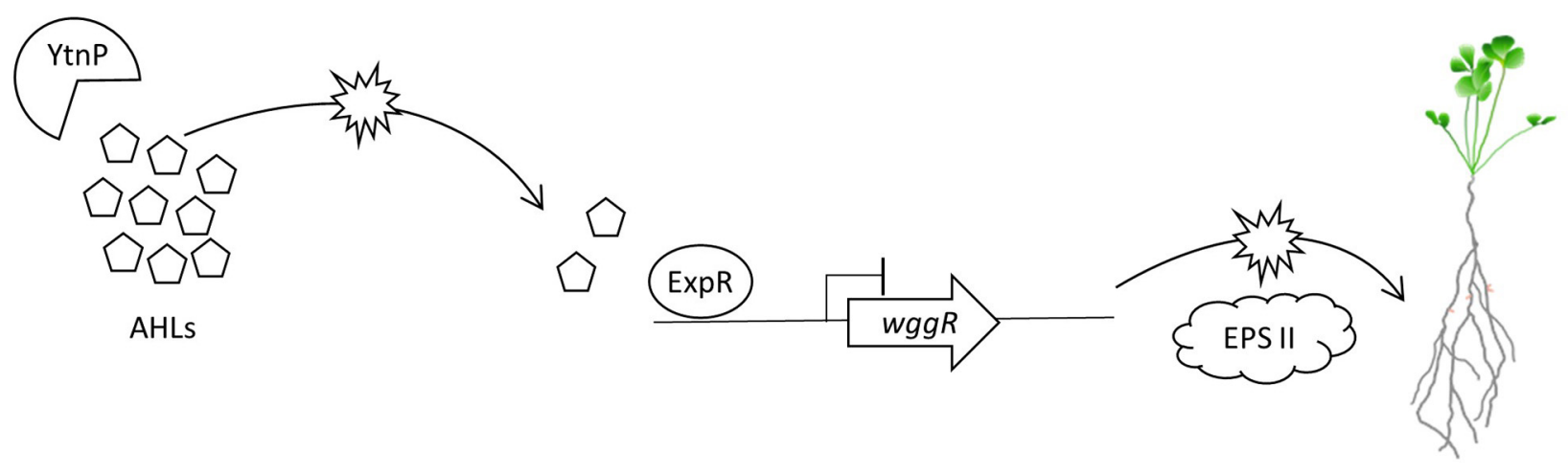

FIGURE 7 | Model of proposed molecular QS and QQ interactions between M. truncatula PGPRs. B. subtilis UD1022 produces the lactonase YtnP which cleaves S. meliloti Rm8530 AHLs. Through quorum quenching, UD1022 YtnP reduces AHL concentrations, inhibiting the upregulation of symbiotically active EPS II genes. This may result in lower nodulation efficiency of Rm8530 in the presence of the PGPR UD1022.

been utilized to identify QQ microbial isolates for this purpose (Tang et al., 2013; Last et al., 2016; Stein and Schikora, 2018). To better understand the capacity of UD1022 for QQ, we used the bioreporter strain Agrobacterium tumefaciens KYC55 in soft agar to detect both short- and long-chain AHLs. When co-cultures of UD1022 were incubated with $10 \mu \mathrm{M}$ purified 3-oxo-C16-AHL for $24 \mathrm{~h}$ and applied to the bioreporter, expression of KYC55 $\beta$-galactosidase was greatly diminished. This reduction of detectable long-chain AHL demonstrates that UD1022 is capable of QQ activity. Response of the bioreporter to co-cultures of UD1022 with C8-HSL was no different from control treatments.

Many modes of QQ by bacteria have been identified with lactonase hydrolytic enzymes being highly described in Bacillus spp. (Kumar et al., 2015). The B. subtilis NCIB3610 putative lactonase YtnP protein sequence had high similarity to that found in UD1022 (Schneider et al., 2012). The YtnP lactonase is a metallolactamase and was found to target $\gamma$-butyrolactone of Streptomyces griseus. The UD1022 YtnP protein possesses the same hallmark metallohydrolase features of the NCIB3610 YtnP, including the HXHXDH motif, indicating that UD1022 YtnP is also a likely a QQ lactonase. The UD1022 $y \operatorname{tn} P^{-}$ mutant was employed in the AHL biosensor assay to test the role of the specific YtnP QQ activity. Rather than fully abolishing $\mathrm{QQ}$, the partial degradation activity remaining may be due to redundant or multiple lactonase-like genes that continue to be expressed in the single $y \operatorname{tn} P$ mutant. The purified YtnP protein incubated with long- and short-chain AHLs showed clear and efficient QQ activity. Exogenous application of pure YtnP degraded C8-AHL, which was not observed in UD1022 live-cell assays. The possible substrate specificity of the YtnP lactonase protein may be more attributable to enzyme concentration rather than on acyl-chain length of the AHL. Lactonases characterized to date are described as having broad activity against a range of AHL acyl chain lengths and substitutions, though with variable active site affinities (Bergonzi et al., 2018).

QQ in the rhizosphere likely plays a large part in PGPR interactions and, consequently, in plant health outcomes.
The presence of AHL molecules in the rhizosphere have been shown to directly elicit functional and beneficial responses from both non-legumes and legumes (Hartmann et al., 2014; Schikora et al., 2016; Hartmann and Rothballer, 2017). Several studies have employed the use of bacterial QQ lactonases to demonstrate the direct and indirect beneficial activities of AHLs and QS on plants. To identify novel QS-controlled proteins, an S. meliloti 1021 construct expressing the QQ lactonase AiiA was found to be significantly deficient in forming nodule initials within the first $12 \mathrm{~h}$ after inoculation (Gao et al., 2007). Zarkani et al. (2013) showed that S. meliloti producing 3-oxo-C14-AHL increased Arabidopsis thaliana resistance to Pseudomonas syringe pv tomato DC3000, while mutants heterologously expressing the Agrobacterium tumefaciens AttM lactonase did not.

The Rm8530 QS system is required to produce symbiotically active EPS II biofilms (Pellock et al., 2000, 2002; Hoang et al., 2004; Gurich and González, 2009). QS is also important in controlling bacterial cell population density, motility toward the plant root, and switching expression pathways from motility to nodulation (Bahlawane et al., 2008; Gao et al., 2012; Calatrava-Morales et al., 2018). The timing and coordination of these activities are intricately controlled through ExpR/SinI QS, and disruptions or interference through QQ has the potential to affect the efficiency and competency of these pathways. The lack of synergistic effects between Rm8530 and UD1022 may be explained, in part, through the QQ activity of UD1022 YtnP lactonase reducing Rm8530 AHL signal molecule concentration, leading to reduced expression of EPS II biosynthesis genes including $w g g R$, and ultimately resulting in inhibition of efficient nodule initiation on $M$. truncatula roots (Figure 7).

\section{CONCLUSION}

We show in our tri-trophic legume-symbiont-PGPR model system that the PGPR B. subtilis UD1022 does not synergistically 
increase $M$. truncatula plant growth or nodulation by the legume symbiont $S$. meliloti $\mathrm{Rm} 8530$. Though there is no direct growth inhibitory effect between the bacterial strains, indirect interactions contribute to the disruption of plant associative activities by the symbiont. UD1022 affects Rm8530 QS controlled biofilm formation through interference with the QS biosynthesis pathway. Further, UD1022 expresses the QQ lactonase YtnP, which cleaves the specific AHLs required to produce symbiotically active EPS II of Rm8530 biofilms. UD1022 likely delays or fails to promote Rm8530 nodulation through the QQ activity of lactonase YtnP and can inhibit synergistic plant growth promotion.

\section{DATA AVAILABILITY STATEMENT}

The raw data supporting the conclusions of this article will be made available by the authors, without undue reservation.

\section{AUTHOR CONTRIBUTIONS}

AR completed lab work pertaining to microbiology and genetic analysis. AR and HPB conceptualized the idea. PBB designed the experiments for the Bacillus mutants. All authors designed the experiments, edited, and contributed to the final manuscript, and approved for submission.

\section{REFERENCES}

Adesemoye, A. O., Torbert, H. A., and Kloepper, J. W. (2009). Plant growthpromoting rhizobacteria allow reduced application rates of chemical fertilizers. Microb. Ecol. 58, 921-929. doi: 10.1007/s00248-009-9531-y

Aloo, B. N., Makumba, B. A., and Mbega, E. R. (2019). The potential of Bacilli rhizobacteria for sustainable crop production and environmental sustainability. Microbiol. Res. 219, 26-39. doi: 10.1016/j.micres.2018.10.011

Amaya-Gómez, C. V., Hirsch, A. M., and Soto, M. J. (2015). Biofilm formation assessment in Sinorhizobium meliloti reveals interlinked control with surface motility. BMC Microbiol. 15:58. doi: 10.1186/s12866-015-0390-z

Angus, A. A., and Hirsch, A. M. (2013). "Biofilm Formation in the Rhizosphere: Multispecies Interactions and Implications for Plant Growth," in Molecular Microbial Ecology of the Rhizosphere, ed. F. J. de Bruijn (New York: John Wiley \& Sons, Ltd), 701-712. doi: 10.1002/9781118297674.ch66

Arora, N. K., Fatima, T., Mishra, I., and Verma, S. (2020). "Microbe-based Inoculants: Role in Next Green Revolution," in Environmental Concerns and Sustainable Development: Volume 2: Biodiversity, Soil and Waste Management, eds V. Shukla and N. Kumar (Singapore: Springer), 191-246. doi: 10.1007/978981-13-6358-0_9

Bahlawane, C., McIntosh, M., Krol, E., and Becker, A. (2008). Sinorhizobium meliloti regulator MucR couples exopolysaccharide synthesis and motility. Mol. Plant Microbe Interact. MPMI 21, 1498-1509. doi: 10.1094/MPMI-21-11-1498

Bai, Y., Zhou, X., and Smith, D. L. (2003). Enhanced soybean plant growth resulting from coinoculation of Bacillus strains with Bradyrhizobium japonicum. Crop Sci. 43, 1774-1781. doi: 10.2135/cropsci2003.1774

Bartels, F. W., McIntosh, M., Fuhrmann, A., Metzendorf, C., Plattner, P., Sewald, N., et al. (2007). Effector-stimulated single molecule protein-DNA interactions of a quorum-sensing system in Sinorhizobium meliloti. Biophys. J. 92, 43914400. doi: 10.1529/biophysj.106.082016

Baumgardt, K., Charoenpanich, P., McIntosh, M., Schikora, A., Stein, E., Thalmann, S., et al. (2014). RNase E affects the expression of the acylhomoserine lactone synthase gene sinI in Sinorhizobium meliloti. J. Bacteriol. 196, 1435-1447. doi: 10.1128/JB.01471-13

\section{FUNDING}

This work was supported by funding and support from University of Delaware. AR was a DENIN Environmental Fellow and acknowledges support Delaware Environmental Institute (DENIN). HPB acknowledges support from BASF as part of research sponsorship project.

\section{ACKNOWLEDGMENTS}

We thank Dr. Juan E. González for providing S. meliloti strain Rm8530, Dr. Stephen Winans for providing Agrobacterium tumefaciens KYC55 (pJZ410) (pJZ384) (pJZ372), and the University of North Carolina School of Medicine Center for Structural Biology for UD1022 ytnP protein expression. The results, in part, are based on work reported in the master's thesis of Amanda Rosier, which is freely available through the University of Delaware Library Institutional Repository (http://udspace.udel.edu/bitstream/handle/19716/21138/2016_ RosierAmanda_MS.pdf).

\section{SUPPLEMENTARY MATERIAL}

The Supplementary Material for this article can be found online at: https://www.frontiersin.org/articles/10.3389/fmicb. 2020.596299/full\#supplementary-material

Berendsen, R. L., Vismans, G., Yu, K., Song, Y., Jonge, R., de, et al. (2018). Diseaseinduced assemblage of a plant-beneficial bacterial consortium. ISME J. 12:1496. doi: 10.1038/s41396-018-0093-1

Bergonzi, C., Schwab, M., Naik, T., Daudé, D., Chabrière, E., and Elias, M. (2018). Structural and biochemical characterization of AaL, a quorum quenching lactonase with unusual kinetic properties. Sci. Rep. 8:11262. doi: 10.1038/ s41598-018-28988-5

Beringer, J. E. (1974). R factor transfer in Rhizobium leguminosarum. J. Gen. Microbiol. 84, 188-198. doi: 10.1099/00221287-84-1-188

Bishnoi, U., Polson, S. W., Sherrier, D. J., and Bais, H. P. (2015). Draft genome sequence of a natural root isolate, Bacillus subtilis UD1022, a potential plant growth-promoting biocontrol agent. Genome Announc. 3, e696-e615. doi: 10. 1128/genomeA.00696-15

Bogino, P. C., de las Mercedes Oliva, M., Sorroche, F. G., and Giordano, W. (2013). The Role of bacterial biofilms and surface components in plant-bacterial associations. Int. J. Mol. Sci. 14, 15838-15859. doi: 10.3390/ijms140815838

Calatrava-Morales, N., McIntosh, M., and Soto, M. J. (2018). Regulation mediated by $\mathrm{N}$-acyl homoserine lactone quorum sensing signals in the Rhizobium-legume symbiosis. Genes 9:263. doi: 10.3390/genes9050263

Chan, K.-G., Yin, W.-F., and Hong, K.-W. (2016). "Recent Advances in Bacterial Quorum Quenching," in Stress and Environmental Regulation of Gene Expression and Adaptation in Bacteria, ed. F. J. de Bruijn (New York: John Wiley \& Sons, Inc), 1206-1220. doi: 10.1002/9781119004813.ch117

d'Angelo-Picard, C., Faure, D., Penot, I., and Dessaux, Y. (2005). Diversity of $\mathrm{N}$-acyl homoserine lactone-producing and -degrading bacteria in soil and tobacco rhizosphere. Environ. Microbiol. 7, 1796-1808. doi: 10.1111/j.14622920.2005.00886.x

Dong, Y. H., Wang, L. H., Xu, J. L., Zhang, H. B., Zhang, X. F., and Zhang, L. H. (2001). Quenching quorum-sensing-dependent bacterial infection by an N-acyl homoserine lactonase. Nature 411, 813-817. doi: 10.1038/3508 1101

Edgar, R. C. (2004). MUSCLE: multiple sequence alignment with high accuracy and high throughput. Nucleic Acids Res. 32, 1792-1797. doi: 10.1093/nar/ gkh340 
Felici, C., Vettori, L., Giraldi, E., Forino, L. M. C., Toffanin, A., Tagliasacchi, A. M., et al. (2008). Single and co-inoculation of Bacillus subtilis and Azospirillum brasilense on Lycopersicon esculentum: Effects on plant growth and rhizosphere microbial community. Appl. Soil Ecol. 40, 260-270. doi: 10.1016/j.apsoil.2008. 05.002

Fetzner, S. (2015). Quorum quenching enzymes. J. Biotechnol. 201, 2-14. doi: 10.1016/j.jbiotec.2014.09.001

Fox, S. L., O'Hara, G. W., and Bräu, L. (2011). Enhanced nodulation and symbiotic effectiveness of Medicago truncatula when co-inoculated with Pseudomonas fluorescens WSM3457 and Ensifer (Sinorhizobium) medicae WSM419. Plant Soil 348, 245-254. doi: 10.1007/s11104-011-0959-8

Gamalero, E., and Glick, B. R. (2011). "“Mechanisms Used by Plant GrowthPromoting Bacteria,", in Bacteria in Agrobiology: Plant Nutrient Management, ed. D. K. Maheshwari (Berlin: Springer Berlin Heidelberg), 17-46. doi: 10.1007/ 978-3-642-21061-7_2

Gao, M., Chen, H., Eberhard, A., Gronquist, M. R., Robinson, J. B., Connolly, M., et al. (2007). Effects of AiiA-mediated quorum quenching in Sinorhizobium meliloti on quorum-sensing signals, proteome patterns, and symbiotic interactions. Mol. Plant Microbe Interact. MPMI 20, 843-856. doi: 10.1094/ MPMI- 20-7-0843

Gao, M., Chen, H., Eberhard, A., Gronquist, M. R., Robinson, J. B., Rolfe, B. G., et al. (2005). sinI- and $\exp R$-dependent quorum sensing in Sinorhizobium meliloti. J. Bacteriol. 187, 7931-7944. doi: 10.1128/JB.187.23.7931-7944. 2005

Gao, M., Coggin, A., Yagnik, K., and Teplitski, M. (2012). Role of specific quorumsensing signals in the regulation of exopolysaccharide II production within Sinorhizobium meliloti spreading colonies. PLoS One 7:e42611. doi: 10.1371/ journal.pone.0042611

Garcia, J., Barker, D. G., and Journet, E. P. (2006). "Seed Storage and Germination,", in The Medicago truncatula Handbook, ed. U. Mathesius (Ardmore, OK: The Samuel Roberts Noble Foundation).

Glazebrook, J., and Walker, G. C. (1989). A novel exopolysaccharide can function in place of the calcofluor-binding exopolysaccharide in nodulation of alfalfa by Rhizobium meliloti. Cell 56, 661-672. doi: 10.1016/0092-8674(89)90588-6

González, J. E., Reuhs, B. L., and Walker, G. C. (1996). Low molecular weight EPS II of Rhizobium meliloti allows nodule invasion in Medicago sativa. Proc. Natl. Acad. Sci. 93, 8636-8641. doi: 10.1073/pnas.93.16.8636

Gouda, S., Kerry, R. G., Das, G., Paramithiotis, S., Shin, H.-S., and Patra, J. K. (2018). Revitalization of plant growth promoting rhizobacteria for sustainable development in agriculture. Microbiol. Res. 206, 131-140. doi: 10.1016/j.micres. 2017.08.016

Gourion, B., Berrabah, F., Ratet, P., and Stacey, G. (2015). Rhizobium-legume symbioses: the crucial role of plant immunity. Trends Plant Sci. 20, 186-194. doi: $10.1016 /$ j.tplants.2014.11.008

Grandclément, C., Tannières, M., Moréra, S., Dessaux, Y., and Faure, D. (2016). Quorum quenching: role in nature and applied developments. FEMS Microbiol. Rev. 40, 86-116. doi: 10.1093/femsre/fuv038

Guiñazú, L. B., Andrés, J. A., Del Papa, M. F., Pistorio, M., and Rosas, S. B. (2010). Response of alfalfa (Medicago sativa L.) to single and mixed inoculation with phosphate-solubilizing bacteria and Sinorhizobium meliloti. Biol. Fertil. Soils 46, 185-190. doi: 10.1007/s00374-009- 0408-5

Gurich, N., and González, J. E. (2009). Role of quorum sensing in Sinorhizobium meliloti-alfalfa symbiosis. J. Bacteriol. 191, 4372-4382. doi: 10.1128/JB.003 76-09

Halverson, L. J., and Handelsman, J. (1991). Enhancement of soybean nodulation by Bacillus cereus UW85 in the field and in a growth chamber. Appl. Environ. Microbiol. 57, 2767-2770. doi: 10.1128/aem.57.9.2767-2770.1991

Hartmann, A., and Rothballer, M. (2017). "Role of Quorum Sensing Signals of Rhizobacteria for Plant Growth Promotion," in Rhizotrophs: Plant Growth Promotion to Bioremediation, ed. S. Mehnaz (Singapore: Springer Singapore), 205-217. doi: 10.1007/978-981-10-4862-3_10

Hartmann, A., Rothballer, M., Hense, B. A., and Schroder, P. (2014). Bacterial quorum sensing compounds are important modulators of microbe-plant interactions. Front. Plant Sci. 5:131. doi: 10.3389/fpls.2014.00131

Hassan, S., and Mathesius, U. (2012). The role of flavonoids in rootrhizosphere signalling: opportunities and challenges for improving plantmicrobe interactions. J. Exp. Bot. 63, 3429-3444. doi: 10.1093/jxb/err 430
Herridge, D. F., Peoples, M. B., and Boddey, R. M. (2008). Global inputs of biological nitrogen fixation in agricultural systems. Plant Soil 311, 1-18. doi: $10.1007 /$ s11104-008-9668-3

Hintze, J. (2000). NCSS 2000. NCSS, LLC. Kaysville, Utah: USA. www.ncss.com.

Hoang, H. H., Becker, A., and González, J. E. (2004). The LuxR homolog ExpR, in combination with the Sin quorum sensing system, plays a central role in Sinorhizobium meliloti gene expression. J. Bacteriol. 186, 5460-5472. doi: 10. 1128/JB.186.16.5460-5472.2004

Hoang, H. H., Gurich, N., and González, J. E. (2008). Regulation of motility by the ExpR/Sin quorum-sensing system in Sinorhizobium meliloti. J. Bacteriol. 190, 861-871. doi: 10.1128/JB.01310-07

Joelsson, A. C., and Zhu, J. (2005). LacZ-Based Detection of Acyl-Homoserine Lactone Quorum- Sensing Signals. Available online at: http://onlinelibrary.wiley. com/doi/10.1002/9780471729259.mc01c02s3/abstract (accessed on October 10, 2014).

Kang, Y., Shen, M., Yang, X., Cheng, D., and Zhao, Q. (2014). A plant growthpromoting rhizobacteria (PGPR) mixture does not display synergistic effects, likely by biofilm but not growth inhibition. Microbiology 83, 666-673. doi: $10.1134 /$ S0026261714050166

Kloepper, J. W., Leong, J., Teintze, M., and Schroth, M. N. (1980). Enhanced plant growth by siderophores produced by plant growth-promoting rhizobacteria. Nature 286, 885-886. doi: 10.1038/286885a0

Kondorosi, E., Gyuris, J., Schmidt, J., John, M., Duda, E., Hoffmann, B., et al. (1989). Positive and negative control of nod gene expression in Rhizobium meliloti is required for optimal nodulation. EMBO J. 8, 1331-1340. doi: 10. 1002/j.1460-2075.1989.tb03513.x

Koo, B.-M., Kritikos, G., Farelli, J. D., Todor, H., Tong, K., Kimsey, H., et al. (2017). Construction and analysis of two genome-scale deletion libraries for Bacillus subtilis. Cell Syst. 4, 291-305.e. doi: 10.1016/j.cels.2016.12.013

Kumar, P., Koul, S., Patel, S. K. S., Lee, J.-K., and Kalia, V. C. (2015). "Heterologous Expression of Quorum Sensing Inhibitory Genes in Diverse Organisms," in Quorum Sensing vs Quorum Quenching: A Battle with No End in Sight, ed. V. C. Kalia (New Delhi: Springer India), 343-356. doi: 10.1007/978-81-322-19 82-8_28

Last, D., Krüger, G. H. E., Dörr, M., and Bornscheuer, U. T. (2016). Fast, continuous, and high- throughput (bio)chemical activity assay for N-acyl-lHomoserine lactone quorum-quenching enzymes. Appl. Environ. Microbiol. 82, 4145-4154. doi: 10.1128/AEM.00830-16

Lullien, V., Barker, D. G., Lajudie, P., de, and Huguet, T. (1987). Plant gene expression in effective and ineffective root nodules of alfalfa (Medicago sativa). Plant Mol. Biol. 9, 469-478. doi: 10.1007/BF00015878

Marketon, M. M., Glenn, S. A., Eberhard, A., and González, J. E. (2003). Quorum sensing controls exopolysaccharide production in Sinorhizobium meliloti. J. Bacteriol. 185, 325-331. doi: 10.1128/jb.185.1.325-331.2003

Marketon, M. M., and Gonzalez, J. E. (2002). Identification of two quorum-sensing systems in Sinorhizobium meliloti. J. Bacteriol. 184, 3466-3475. doi: 10.1128/JB. 184.13.3466- 3475.2002

Marketon, M. M., Gronquist, M. R., Eberhard, A., and González, J. E. (2002). Characterization of the Sinorhizobium meliloti $\sin R / \sin I$ locus and the production of novel N-acyl homoserine lactones. J. Bacteriol. 184, 5686-5695. doi: 10.1128/jb.184.20.5686-5695.2002

Marrone, P. G. (2019). Pesticidal natural products - status and future potential. Pest Manag. Sci. 75, 2325-2340. doi: 10.1002/ps.5433

Masciarelli, O., Llanes, A., and Luna, V. (2014). A new PGPR co-inoculated with Bradyrhizobium japonicum enhances soybean nodulation. Microbiol. Res. 169, 609-615. doi: 10.1016/j.micres.2013.10.001

Maymon, M., Martínez-Hidalgo, P., Tran, S. S., Ice, T., Craemer, K., Anbarchian, T., et al. (2015). Mining the phytomicrobiome to understand how bacterial coinoculations enhance plant growth. Front. Plant Sci. 6:784. doi: 10.3389/fpls. 2015.00784

McIntosh, M., Meyer, S., and Becker, A. (2009). Novel Sinorhizobium meliloti quorum sensing positive and negative regulatory feedback mechanisms respond to phosphate availability. Mol. Microbiol. 74, 1238-1256. doi: 10.1111/j.13652958.2009.06930.x

Meade, H. M., Long, S. R., Ruvkun, G. B., Brown, S. E., and Ausubel, F. M. (1982). Physical and genetic characterization of symbiotic and auxotrophic mutants of Rhizobium meliloti induced by transposon Tn5 mutagenesis. J. Bacteriol. 149:114. doi: 10.1128/jb.149.1.114-122.1982 
Morel, M. A., Cagide, C., Minteguiaga, M. A., Dardanelli, M. S., and CastroSowinski, S. (2015). The pattern of secreted molecules during the co-inoculation of alfalfa plants with Sinorhizobium meliloti and Delftia sp. strain JD2: an interaction that improves plant yield. Mol. Plant Microbe Interact. MPMI 28, 134-142. doi: 10.1094/MPMI-08-14-0229-R

Morel, M. A., Ubalde, M. C., Braña, V., and Castro-Sowinski, S. (2011). Delftia sp. JD2: a potential $\mathrm{Cr}(\mathrm{VI})$-reducing agent with plant growth-promoting activity. Arch. Microbiol. 193, 63-68. doi: 10.1007/s00203-010-0632-2

Oldroyd, G. E. D. (2013). Speak, friend, and enter: signalling systems that promote beneficial symbiotic associations in plants. Nat. Rev. Microbiol. 11, 252-263. doi: 10.1038/nrmicro2990

Oldroyd, G. E. D., Murray, J. D., Poole, P. S., and Downie, J. A. (2011). The rules of engagement in the legume-rhizobial symbiosis. Annu. Rev. Genet. 45, 119-144. doi: 10.1146/annurev-genet- 110410-132549

O’Toole, G., Kaplan, H. B., and Kolter, R. (2000). Biofilm formation as microbial development. Annu. Rev. Microbiol. 54, 49-79. doi: 10.1146/annurev.micro.54. 1.49

O’Toole, G. A., Pratt, L. A., Watnick, P. I., Newman, D. K., Weaver, V. B., and Kolter, R. (1999). “"'Genetic approaches to study of biofilms,", in Methods in Enzymology Biofilms, ed. R. J. Doyle (New York: Academic Press), 91-109. doi: 10.1016/s0076-6879(99)10008-9

Pellock, B. J., Cheng, H.-P., and Walker, G. C. (2000). Alfalfa root nodule invasion efficiency is dependent on Sinorhizobium meliloti polysaccharides. J. Bacteriol. 182, 4310-4318. doi: 10.1128/jb.182.15.4310-4318.2000

Pellock, B. J., Teplitski, M., Boinay, R. P., Bauer, W. D., and Walker, G. C. (2002). A LuxR homolog controls production of symbiotically active extracellular polysaccharide II by Sinorhizobium meliloti. J. Bacteriol. 184, 5067-5076. doi: 10.1128/JB.184.18.5067- 5076.2002

Peoples, M. B., Brockwell, J., Herridge, D. F., Rochester, I. J., Alves, B. J. R., Urquiaga, S., et al. (2009). The contributions of nitrogen-fixing crop legumes to the productivity of agricultural systems. Symbiosis 48, 1-17. doi: 10.1007/ BF03179980

Peters, N. K., Frost, J. W., and Long, S. R. (1986). A plant flavone, luteolin, induces expression of Rhizobium meliloti nodulation genes. Science 233, 977-980. doi: $10.1126 /$ science. 3738520

Pii, Y., Mimmo, T., Tomasi, N., Terzano, R., Cesco, S., and Crecchio, C. (2015). Microbial interactions in the rhizosphere: beneficial influences of plant growthpromoting rhizobacteria on nutrient acquisition process. Review. Biol. Fertil. Soils 51, 403-415. doi: 10.1007/s00374-015-0996-1

Rinaudi, L. V., and Gonzalez, J. E. (2009). The low-molecular-weight fraction of exopolysaccharide II from Sinorhizobium meliloti is a crucial determinant of biofilm formation. J. Bacteriol. 191, 7216-7224. doi: 10.1128/JB.01063-09

Rodríguez, M., Torres, M., Blanco, L., Béjar, V., Sampedro, I., and Llamas, I. (2020). Plant growth- promoting activity and quorum quenching-mediated biocontrol of bacterial phytopathogens by Pseudomonas segetis strain P6. Sci. Rep. 10:4121. doi: 10.1038/s41598-020-61084- 1

Rosier, A. A. (2016). An investigation of the tri-trophic interactions in the rhizosphere of Medicago truncatula using a functional microbiome approach. Ph. D. master's thesis. Newark, DE: University of Delaware.

Ryan, P. R., Dessaux, Y., Thomashow, L. S., and Weller, D. M. (2009). Rhizosphere engineering and management for sustainable agriculture. Plant Soil 321, 363383. doi: 10.1007/s11104-009-0001-6

SAS Institute Inc (1989-2019). JMP ${ }^{\circledR}$, Version Pro 14. Cary, NC: SAS Institute Inc, 1989-2019.

Schikora, A., Schenk, S. T., and Hartmann, A. (2016). Beneficial effects of bacteriaplant communication based on quorum sensing molecules of the $\mathrm{N}$-acyl homoserine lactone group. Plant Mol. Biol. 90, 605-612. doi: 10.1007/s11103016-0457-8

Schlatter, D. C., Bakker, M. G., Bradeen, J. M., and Kinkel, L. L. (2015). Plant community richness and microbial interactions structure bacterial communities in soil. Ecology 96, 134-142. doi: 10.1890/13-1648.1

Schmittgen, T. D., and Livak, K. J. (2008). Analyzing real-time PCR data by the comparative C(T) method. Nat. Protoc. 3, 1101-1108. doi: 10.1038/nprot. 2008.73
Schneider, J., Yepes, A., Garcia-Betancur, J. C., Westedt, I., Mielich, B., and López, D. (2012). Streptomycin-induced expression in Bacillus subtilis of YtnP, a lactonase-homologous protein that inhibits development and streptomycin production in Streptomyces griseus. Appl. Environ. Microbiol. 78, 599-603. doi: 10.1128/AEM.06992-11

Schwartz, A. R., Ortiz, I., Maymon, M., Herbold, C. W., Fujishige, N. A., Vijanderan, J. A., et al. (2013). Bacillus simplex-a little known PGPB with antifungal activity-alters pea legume root architecture and nodule morphology when coinoculated with Rhizobium leguminosarum bv. viciae. Agronomy 3, 595-620. doi: 10.3390/agronomy3040595

Sergaki, C., Lagunas, B., Lidbury, I., Gifford, M. L., and Schäfer, P. (2018). Challenges and approaches in microbiome research: from fundamental to applied. Front. Plant Sci. 9:1205. doi: 10.3389/fpls.2018.01205

Stagnari, F., Maggio, A., Galieni, A., and Pisante, M. (2017). Multiple benefits of legumes for agriculture sustainability: an overview. Chem. Biol. Technol. Agric. 4:2. doi: 10.1186/s40538- 016-0085-1

Stein, E., and Schikora, A. (2018). Detection of bacterial quorum sensing molecules. Methods Mol. Biol. Clifton N. J. 1734, 171-179. doi: 10.1007/978-1-4939-7604$1 \_14$

Tamura, K., Peterson, D., Peterson, N., Stecher, G., Nei, M., and Kumar, S. (2011). MEGA5: molecular evolutionary genetics analysis using maximum likelihood, evolutionary distance, and maximum parsimony methods. Mol. Biol. Evol. 28, 2731-2739. doi: 10.1093/molbev/msr121

Tang, K., Zhang, Y., Yu, M., Shi, X., Coenye, T., Bossier, P., et al. (2013). Evaluation of a new high- throughput method for identifying quorum quenching bacteria. Sci. Rep. 3:2935. doi: 10.1038/srep02935

Timmusk, S., Behers, L., Muthoni, J., Muraya, A., and Aronsson, A.-C. (2017). Perspectives and challenges of microbial application for crop improvement. Front. Plant Sci. 8:49. doi: 10.3389/fpls.2017.00049

Trabelsi, D., Pini, F., Aouani, M. E., Bazzicalupo, M., and Mengoni, A. (2009). Development of real- time PCR assay for detection and quantification of Sinorhizobium meliloti in soil and plant tissue. Lett. Appl. Microbiol. 48, 355361. doi: 10.1111/j.1472-765X.2008.02532.x

Tram, G., Korolik, V., and Day, C. J. (2013). MBDS solvent: an improved method for assessment of biofilms. Adv. Microbiol. 03, 200-204. doi: 10.4236/aim.2013. 32030

Yasbin, R. E., and Young, F. E. (1974). Transduction in Bacillus subtilis by bacteriophage SPP1. J. Virol. 14, 1343-1348. doi: 10.1128/jvi.14.6.1343-1348. 1974

Zarkani, A. A., Stein, E., Röhrich, C. R., Schikora, M., Evguenieva-Hackenberg, E., Degenkolb, T., et al. (2013). Homoserine lactones influence the reaction of plants to Rhizobia. Int. J. Mol. Sci. 14, 17122-17146. doi: 10.3390/ ijms140817122

Zhu, J., Chai, Y., Zhong, Z., Li, S., and Winans, S. C. (2003). Agrobacterium bioassay strain for ultrasensitive detection of $\mathrm{N}$-acylhomoserine lactonetype quorum-sensing molecules: detection of autoinducers in Mesorhizobium huakuii. Appl. Environ. Microbiol. 69, 6949-6953. doi: 10.1128/AEM.69.11. 6949-6953.2003

Zilber-Rosenberg, I., and Rosenberg, E. (2008). Role of microorganisms in the evolution of animals and plants: the hologenome theory of evolution. FEMS Microbiol. Rev. 32, 723-735. doi: 10.1111/j.1574-6976.2008.00123.x

Zipfel, C., and Oldroyd, G. E. D. (2017). Plant signalling in symbiosis and immunity. Nature 543, 328-336. doi: 10.1038/nature22009

Conflict of Interest: The authors declare that the research was conducted in the absence of any commercial or financial relationships that could be construed as a potential conflict of interest.

Copyright (c) 2021 Rosier, Beauregard and Bais. This is an open-access article distributed under the terms of the Creative Commons Attribution License (CC BY). The use, distribution or reproduction in other forums is permitted, provided the original author(s) and the copyright owner(s) are credited and that the original publication in this journal is cited, in accordance with accepted academic practice. No use, distribution or reproduction is permitted which does not comply with these terms. 